\title{
La mirada emergente: el discurso de los procesos de integración
}

\author{
Antonio Trinidad Requena \\ Universidad de Granada. Departamento de Sociología \\ atrinida@ugr.es
}

Recibido: 15-04-2010

Aceptado: 02-06-2010

\section{Resumen}

En el Estado español, las políticas de integración social de los inmigrantes forman parte de las competencias de las comunidades autónomas. Por tal motivo, éstas han diseñado un conjunto de medidas de carácter integral que han puesto en marcha en los últimos años con el nombre de Planes de Integración de los Inmigrantes. Planes que se inscriben en un determinado modelo de integración, lo que podría propiciar la emergencia de importantes diferencias regionales en esta materia que provocarían situaciones heterogéneas de integración. Para conocer la realidad de cada comunidad autónoma, se ha de articular la investigación que se recoge en este artículo de acuerdo con los siguientes objetivos: en primer lugar, estudiar el modelo de integración de que parte cada comunidad autónoma española a la hora de diseñar su política sobre integración de inmigrantes. En segundo lugar, conocer la articulación de los discursos de los destinatarios de esas políticas sobre su proceso de convivencia en cada comunidad. En tercer lugar, estudiar las estrategias sociales seguidas por este grupo para adaptarse a la nueva situación social emergida tras la inmigración.

Palabras clave: inmigrantes; España; integración social; políticas de integración social; convivencia; redes sociales.

\section{Abstract. The emerging look: the discourse of the integration processes}

In Spain, social integration policies for immigrants fall under the powers of the Autonomous Communities. That is why they have developed an integral set of actions that have been implemented over recent years under the name Immigrant Integration Plans, each one of which has a particular integration model. This could lead to important regional differences on this matter, which could provoke heterogeneous integration situations. In order to discover the reality in each Autonomous Community, the research gathered in this article was developed in accordance with the following objectives: first, to study the integration model used by each Autonomous Community in designing its immigrant integration policy; second, to know the opinions of the people addressed by these policies as regards their coexistence process in each Community; third, the social strategies used by this group in order to adapt to the new social situation encountered after immigrating were studied.

Key words: immigrants; Spain; social integration; social integration policy; coexistence; social networks. 


\title{
Sumario
}

\author{
Introducción \\ 4. El reconocimiento legal: \\ 1. Objetivos y diseño metodológico \\ de la investigación \\ el discurso de los "papeles" \\ 5. El papel de las redes sociales en el proceso \\ de integración: «a la gente que conozco» \\ 2. El concepto de integración \\ en los planes autonómicos \\ 3. La integración social: \\ «La metáfora de la camisa» \\ A modo de conclusión \\ Referencias bibliográficas
}

\section{Introducción}

El creciente movimiento de personas entre distintos países provoca un tránsito de culturas, que antes se encontraban circunscritas a un territorio particular. Estos flujos se cruzan en espacios físicos y momentos históricos determinados, lo cual da origen a nodos o puntos de encuentro donde se cruzan elementos culturales, económicos y sociales. En términos dramaturgicos, nos encontramos con un único escenario y con actores entrenados para obras distintas. Ante esta nueva situación, el director tiene tres alternativas: una, cambiar de obra e iniciar nuevos ensayos; dos, mantener la obra e ir incorporando a los nuevos actores, y tres, representar distintas obras en el mismo escenario. Sirva la metáfora para introducir el término integración, tan utilizado cuando nos referimos a inmigración y que es el objeto de este trabajo.

Fue Hannah Arendt, con la publicación, en la década de 1950, de dos libros ya clásicos, Los orígenes del totalitarismo y La condición humana, la que nos advierte del riesgo inherente a la exclusión social. Toda su obra es un alegato en contra de la ausencia de identidad de aquellos que se quedan al margen y que conforman una masa sin identidad conjunta dentro del ideario colectivo para su mejor maleabilidad y control social. Dicha pensadora escribía: «Las masas carecen de esa clase específica de diferenciación» (Arendt, 1993).

Para eludir el riesgo, del que habla Hannah Arendt, los poderes públicos articulan un conjunto de medidas bajo la denominación de "política de integración", que hace referencia al modo que, desde el punto de vista político, jurídico y/o administrativo, se articula para la consecución de dicho fin. Mucho se ha debatido acerca de la conceptualización del término integración, al conformarse como un concepto complejo y con múltiples interpretaciones. Según el diccionario de María Moliner, integración es «la acción de integrar». Y, en integrar, hay tres acepciones: la primera, "hacer un todo o conjunto con partes diversas", seria el modelo de integración multicultural; la segunda, "contribuir a formar un todo o conjunto", se correspondería con el modelo de integración intercultural, y la tercera, "hacer entrar una cosa en otra más amplia», tendría un símil en el modelo de integración asimilacionista.

En consecuencia, con la acción de integrar, la política que pretenda tal fin puede optar por una u otra acepción del término. En el caso español, la polí- 
tica de integración se desarrolla a través de cuatro ámbitos competenciales: el comunitario, el estatal, el autonómico y el local. La pregunta parece obligada: ¿Cuáles son las competencias, las atribuciones y los discursos de cada uno de estos niveles en materia de integración social para los inmigrantes?

En lo que respecta a la Unión Europea, si bien en 1999 los jefes de estado y de gobierno europeos decidieron, en Tampere, impulsar una política común de inmigración y asilo, no será hasta 2003 cuando, en Salónica, el Consejo Europeo dictamine lo siguiente: «[...] es necesario elaborar una política global y multidimensional sobre la integración de las personas (inmigrantes). Teniendo en cuenta que una integración satisfactoria contribuye a la cohesión social y al bienestar económico, esta política debería cubrir factores como el empleo, la participación económica, la educación, la enseñanza de la lengua, servicios sociales y de salud, vivienda y asuntos urbanos, así como la cultura y la participación en la vida social».

Con posterioridad al año 2003, serán los principios básicos comunes sobre integración, aprobados por la Comisión Europea junto con el Fondo Europeo para la Integración y el nuevo Tratado de Lisboa, los principales instrumentos con los que cuenta la Unión Europea para impulsar una política de integración. No obstante, aunque se trata de instrumentos necesarios, no son suficientes. La razón obedece al "carácter no vinculante de los principios y a la cláusula de que es a los Estados a quien corresponde decidir sobre qué políticas de integración desarrollar» (López Pich, 2007: 249). A pesar de ello, lo que sí parece existir es un acuerdo en torno a lo que significa la integración para los estados miembros, y se ofrece un discurso único en cuanto al modelo que se pretende adoptar. Concretamente, se parte de la idea de que la integración es un «proceso bidireccional y dinámico, de ajuste mutuo por parte de todos los inmigrantes y residentes de los Estados miembros».

Por su parte, la regulación del estatuto de derechos sociales de los extranjeros en el ámbito nacional (que se encuentra vinculado a dos ámbitos competenciales diferentes: extranjería y derechos sociales) ${ }^{1}$ adquiere singularidad propia en España, como consecuencia de la implantación del Estado de las Autonomías. El Estado central «al amparo de los distintos títulos competenciales (principalmente, los previstos en los artículos 149.1.1 y 149.1.2 CE), incluyó en la Ley de extranjería 4/2000 un catálogo de los derechos reconocidos a los extranjeros, incluidos los derechos sociales, cuya prestación y competencias corresponden fundamentalmente a las comunidades autónomas» (Manrubia, 2007: 188). Derechos mantenidos en la Ley 2/2009 que reforma la ya citada ley de extranjería 4/2000.

Las omunidades autónomas, con el objetivo de integrar, coordinar y sistematizar las medidas de integración para las personas inmigrantes, y en el marco de la descentralización de competencias, han elaborado y aprobado

1. Sin embargo, en la nueva Ley $2 / 2009$, se han transferido algunas de las competencias estatales a las comunidades autónomas, como consecuencia de los cambios que se han producido en algunos estatutos de autonomía. 
planes autonómicos para la inmigración (o planes para la integración social de las personas inmigrantes, según la denominación en cada caso), acompañados de diferentes diseños institucionales para su implementación. En esta investigación, nos centraremos en cinco de las comunidades con mayor proporción de población inmigrada. De un lado, trataremos de estudiar el concepto de integración plasmado en sus respectivos Planes autonómicos, para intentar averiguar si podemos hablar de algún tipo de modelo español propio de integración, al igual que en el mundo conceptual existen los modelos inglés, francés o alemán. De otro lado, daremos la palabra a los destinatarios de esas políticas, los inmigrantes, para conocer cómo interpretan, dan sentido y articulan su discurso sobre la integración y su proceso en cada comunidad autónoma.

\section{Objetivos y diseño metodológico de la investigación}

Los estudios españoles sobre las migraciones han tenido un impulso importante en los últimos años, a pesar de la reciente incorporación de la sociología española al estudio de tal fenómeno social. Llopis agrupa los estudios sociológicos sobre inmigración en dos grupos. Un primer grupo concentraría aquellos dirigidos a estudiar las actitudes y las opiniones de la población española ante la población inmigrante. Un segundo grupo aglutinaría «aunque en una medida muy inferior, aquellos estudios destinados a investigar el proceso de integración de la propia población inmigrante» (Llopis, 2007: 192-193). Pues bien, es precisamente en este segundo grupo donde se inserta la presente investigación. Investigación que se enmarca en un proyecto más amplio, puesto que aquí solo nos centramos en uno de sus objetivos, financiado por el Ministerio de Ciencia e Innovación en la convocatoria de proyectos de I+D, en la edición del 2006, bajo el título Bienestar social e inmigración: Las politicas de integración en las Comunidades Autónomas españolas (SEJ 2006-05222). Proyecto centrado en dar respuesta a una serie de interrogantes, por ejemplo: «QQué política de integración sigue cada comunidad autónoma?», «En el caso de ser distinta, ‘̨a qué modelo de integración responde cada una?», «¿Son estas políticas las más adecuadas para nuestro país?» y «¿Cómo valora el inmigrante las distintas actuaciones públicas?».

Interrogantes que nos llevan a plantear la siguiente hipótesis de partida: en el Estado español, las políticas de integración social de los inmigrantes forman parte de las competencias de las comunidades autónomas y, en cada una de ellas, es posible detectar modelos de intervención, planificación y gestión diferentes. El resultado podría ser la posible emergencia de importantes diferenciaciones regionales en esta materia. Diferenciaciones que provocarían situaciones heterogéneas de integración. Para dar respuesta a las preguntas planteadas y confirmar o anular la hipótesis, se hace necesario articular la investigación de acuerdo con los siguientes objetivos. En primer lugar, estudiar el modelo de integración de que parte cada comunidad autónoma española a la hora de diseñar su política sobre integración de inmigrantes. En segundo lugar, conocer la articulación de los discursos de los destinatarios de esas políticas sobre su proceso de convivencia en cada comunidad. 
Para conseguir los objetivos que han guiado esta investigación, se ha utilizado una metodología de carácter cualitativo, ya que la pretensión del trabajo era conocer la percepción subjetiva de los inmigrantes sobre su idea de integración y saber las estrategias sociales que se deben utilizar en el camino de la regularización de su situación administrativa. En definitiva, dar la palabra a los actores para que nos enseñen su realidad, que, en ocasiones, no coincide con la que emerge de la interpretación de los datos cuantitativos. Con el fin de conocer el discurso de los inmigrantes, se ha utilizado la entrevista abierta como técnica de producción de datos.

El diseño de la muestra se ha realizado partiendo de unos perfiles teóricos de grupos que, en principio, se creía iban a determinar los discursos de los inmigrantes. Los distintos perfiles elaborados intentan responder, con el mayor rigor científico posible, a la heterogeneidad de la población inmigrante. En definitiva, se han tenido en cuenta cuatro características fundamentalmente: ámbito de vida — comunidad autónoma—, país de origen, nivel de estudios y, por último, género. Estas cuatro dimensiones, en mayor o menor medida, explican parte de los discursos de las personas que han participado en la investigación. En esta investigación, nos centraremos en cinco de las comunidades con mayor proporción de población inmigrada: Andalucía, Cataluña, Comunidad Valenciana, Comunidad de Madrid y Región de Murcia.

Del análisis de los discursos de los inmigrantes, se ha llegado a los siguientes resultados, estructurados en cuatro apartados o epígrafes. En el primer epígrafe, se analiza el concepto de integración en los distintos planes de integración. En el segundo, se reconstruye el discurso sobre la idea de integración en un grupo social tan heterogéneo como el de los inmigrantes, donde emerge una nueva mirada social, ocupada por la percepción subjetiva y objetiva de la desigualdad social. En el tercero, se estudian las estrategias sociales seguidas por este grupo para adaptarse a la nueva situación social emergida tras la inmigración, y, a continuación, el peso que tienen las redes sociales en todo el proceso de integración social. Por último, se sintetizan las conclusiones derivadas de la investigación.

\section{El concepto de integración en los planes autonómicos}

Cuando las migraciones son estudiadas desde el país de acogida de los inmigrantes, se plantean una serie de cuestiones y problemas que no tienen fácil solución. Las autoridades competentes han de jugar y mantener el equilibrio de una balanza frágil e inestable. Por una parte, en el caso de España, la llegada de inmigrantes se vislumbra como una posible solución al envejecimientode la población y al mantenimiento del Estado de bienestar. Por otro lado, dentro de la pluralidad de valores, de naciones o pueblos (según los recientes debates), de la cultura de cada país, etc., hay cierta preocupación por el mantenimiento de una identidad basada en el respeto de los valores democráticos y de los derechos humanos. En definitiva, se trata de articular el proceso de integración o convivencia cívica.

Según el Diccionario enciclopédico de sociología, de Karl-Heinz Hillmann (2001), la sociología nos dice que «la integración es la denominación con que 
se conoce en sociología a los procesos de incorporación y asimilación, en la conciencia y en la práctica, de las estructuras de valor y las pautas de conducta llevadas a cabo: a) por personas individuales en relación con determinados grupos $\mathrm{u}$ organizaciones, o sectores de la sociedad relevantes para ellas; $b$ ) entre distintos grupos, clases o etnias de una sociedad; $c$ ) entre distintas sociedades, en beneficio de la constitución de estructuras y órdenes sociales culturales comunes, nuevos».

Pero el problema de tal proceso radica en la posición que cada uno ocupa, según el caso, en las estructuras de poder para decidir entre incorporación en igualdad de condiciones o asimilación. Sartori (2006) mantiene que un multiculturalismo extremista valora más la distancia cultural con el inmigrante que las similitudes mutuas en emociones, deseos e intereses, mientras el interculturalismo sí plantea la necesidad de fomentar dichas identificaciones, lo cual provoca un acercamiento y no un distanciamiento. Por tanto, las posiciones son múltiples y defendidas desde espacios sociales de desigualdad. Ariño (2007) señala que éstas se centran fundamentalmente en cuatro:

1) El modelo asimilacionista, que sostiene que el pluralismo cultural debe subordinarse a la aceptación de los valores propios del consenso liberal en torno a los derechos individuales (Sartori, 2006).

2) El modelo multicultural, donde se defiende la viabilidad del reconocimiento de los derechos de las identidades colectivas, cada una de las cuales vive inmersa en su nicho. Se maneja un concepto esencialista de cultura (Australia y Canadá).

3) El modelo segregación generalizada parte de que el choque es inevitable, puesto que unas culturas son incompatibles con otras. Conduce al apartheid cultural (Huntington, 2004).

4) El modelo pragmático, donde se defiende que los científicos sociales deben estudiar los contextos reales, las interacciones cotidianas y los procesos de negociación y mediación que se producen en ellos.

Por tanto, aunque la convivencia pueda ser precaria y contingente, se fortalece cuando se asienta sobre procedimientos regulados de arbitraje, mediación y espacios de convergencia (Bauman, 2001).

La clave para alcanzar un equilibrio entre las consideraciones planteadas radica en la correcta implementación de políticas públicas que permitan la integración social de los inmigrantes, a la vez que los que llegan respetan y aceptan unos valores mínimos por los que se rige la sociedad que les acoge, lo cual permite la estabilidad del sistema y el justo intercambio de intereses; en definitiva, una convivencia cívica. Como señala Karl-Heinz Hillmann (2001), la integración o la incorporación de los trabajadores inmigrantes es problemática y, en parte, es conflictivo el grado de predisposición de los inmigrantes a integrarse culturalmente, aparte de las muy diversas actitudes que la población autóctona (desde la tolerancia hasta el racismo) adopta ante ellos.

Las políticas públicas dirigidas a la integración de los inmigrantes se han ido conformando en dos ámbitos competenciales: «por un lado, la situación 
jurídico-administrativa del inmigrante, de exclusiva competencia estatal; por otro, su integración social, en la que destacan, aunque no de forma exclusiva, las competencias autonómicas» (Montilla Martos, 2006: 341). Así, las comunidades autónomas se comprometen a asegurar la prestación de servicios sociales básicos, lo que les ha llevado a establecer un conjunto de medidas de carácter integral que la mayoría de ellas han puesto en marcha en los últimos años en forma de planes de integración de los inmigrantes.

El impulso para la creación de estos planes vino dado por el Plan para la Integración Social de los Inmigrantes (PISI), aprobado el 2 de diciembre de 1994 por el Consejo de Ministros. Tras el PISI, la siguiente medida que ha influido en las políticas de integración ha sido el Programa Global de Regulación y Coordinación de Extranjería e Inmigración (GRECO), aprobado en 2001 y sustituido por el actual Plan Estratégico de Ciudadanía e Integración.

Al ser el Plan Estratégico de Ciudadanía e Integración el nuevo marco en el que se insertan y se coordinan todas las políticas de inmigración, tanto a nivel estatal como regional, resulta relevante ver cuál es el concepto de integración que se define en el PECI y en cada una de las comunidades autónomas (Pérez Yruela y Rinken, 2005; Solé, 2002). En el PECI, por ejemplo, el modelo o concepto de integración utilizado es el referido en los Principios básicos comunes para las políticas de integración de los inmigrantes en la Unión Europea $(2004)^{2}$ y en los que se define la integración como «un proceso bidireccional y dinámico de ajuste mutuo por parte de todos los inmigrantes y residentes de los Estados miembros», que se caracteriza por «implicar el respeto de los valores básicos de la UE». Sin embargo, como señala Soriano (2010), en la última reforma de la Ley de extranjería 2/2009, se aleja de la bidireccionalidad por la que apuesta el PECI y se apuesta por una integración coercitiva.

En el trasfondo de este trabajo, una de las preguntas que se deben responder versa sobre los diferentes modelos de integración que se desarrollan en cada comunidad autónoma. Repasando cada uno de los planes, se encuentran definiciones claras y extensas del modelo de integración que intentará desarrollar cada una, aunque también hay comunidades que no tratan dicha cuestión y pasan directamente a la elaboración de medidas encaminadas a un modelo de integración que se da por supuesto. La mayoría de los planes comienzan con un preámbulo o una presentación donde quedan establecidas las líneas generales del plan, el trasfondo ideológico que lo articula y las pretensiones de cada gobierno regional. Por eso, dichos preámbulos son a veces firmados por el presidente autonómico o por el máximo responsable de las políticas de inmigración de la comunidad, aunque en otras ocasiones se trata de una exposición resumida de lo que va a ser el plan. Sirva como ejemplo el somero análisis que se realiza de los preámbulos de los planes que se exponen a continuación.

En el Plan de Andalucía, la política de inmigración arranca de una visión integral del fenómeno, cuyo fin último es el de la plena incorporación de la per-

2. Estos principios fueron aprobados por el Consejo de la Unión Europea y los representantes de los gobiernos de los estados miembros el 19 de noviembre de 2004. 
sona inmigrante a la sociedad receptora. La integración está basada en el principio de normalización, con lo que se intenta evitar la duplicidad de sistemas sociales y el peligro de fomentar la exclusión o la marginación de ciertos grupos. Se prevé el refuerzo de los servicios ordinarios en caso de ser necesario para favorecer la integración gradual. Se reconoce la bidireccionalidad del proceso y la importancia de llevar a la práctica medidas que mejoren la sensibilización y aporten una visión positiva de la inmigración entre la población de acogida, a la vez que se destaca la posibilidad de que se produzca un enriquecimiento mutuo y continuo.

En el caso de Cataluña, el modelo de integración queda definido al hablar del modelo de ciudadanía que se quiere alcanzar, una ciudadanía plural y cívica. Se apuesta por un modelo de sociedad basado en la igualdad, con un proyecto cultural y político, abierto y plural. También en Cataluña se reconoce el carácter bidireccional del proceso de integración, con lo cual se confirma que una plena integración de los inmigrantes requiere esfuerzo por ambas partes e incluso renuncias, pero, a la vez, un sinfín de nuevas oportunidades. Se mantiene como interrogante si realmente la inmigración aporta a la sociedad más de lo que recibe, y si los pilares del Estado de bienestar se fortalecerán con estos cambios o si, por el contrario, se tambalearán aún más.

El nuevo Plan de Madrid no define el concepto de integración al que se acoge, pero sí se amplía una escueta definición con una enumeración de principios que también permiten conocer realmente qué modelo de integración guía a las medidas propuestas. Se enumeran como principios rectores los siguientes: la cohesión social, la igualdad de oportunidades, la corresponsabilidad, la normalización, la interculturalidad y la coordinación. De todos estos principios, el que más interesa para describir el modelo de integración de que se trata es el principio de interculturalidad, que viene detallado de la siguiente manera: «Las sociedades modernas actuales se van haciendo más complejas con la incorporación de personas de culturas, etnias e identidades distintas y plurales a las preexistentes en ellas. Por esa razón, el horizonte debe abrirse no solamente a la pluralidad cultural, sino también a las nuevas formas sociales y culturales, que son fruto de los procesos de intercambio tan fructíferos entre los que ya están y los que llegan ahora. La interculturalidad supone el reconocimiento de unos y de otros como distintos pero iguales que conviven y comparten no solamente espacios comunes, sino también proyectos comunes». A través de estas líneas, se vislumbra una superación del modelo pluricultural, que, a pesar de presentarse como idóneo para sociedades como la europea, ha dado muestras de ser ineficaz para conseguir una auténtica integración de los inmigrantes ${ }^{3}$, ya que genera situaciones de inestabilidad.

3. Blanco (2000): «De cualquier modo, el pluralismo cultural es más un ideal que una realidad, y si bien las minorías y la sociedad principal tienden a mantener sus culturas, esto produce constantemente conflictos importantes en el seno de las sociedades multiculturales. El pluralismo cultural es, hoy por hoy, una situación de equilibrio inestable en la mayoría de las sociedades receptoras». 
Sin duda alguna, es en el Plan de Murcia donde el concepto de integración es definido desde la primera página a raíz del siguiente fragmento: «[...] no es posible desarrollar un enfoque integrado respecto a la inmigración sin considerar el impacto de las políticas de migración en la sociedad de acogida y en los propios migrantes. Las condiciones sociales a que se enfrentan los migrantes, la actitud de la población de acogida y la presentación, por parte de los responsables políticos, de los beneficios de la diversidad y de las sociedades pluralistas, son vitales para el éxito de las políticas de inmigración» ${ }^{4}$.

En este plan, la integración conlleva la bidireccionalidad. Se trata de un cambio de mentalidad y de comportamientos, «no por sustracción sino por adición», es decir, la integración supondrá más enriquecimiento que empobrecimiento, será más positiva que negativa, aportará más de lo que consume. Se propone un modelo que no quiere imponer ni asimilar, aunque tampoco eso suponga renunciar a lo propio. "Consiste en convivir interculturalmente, enriqueciéndonos mutuamente con las aportaciones del otro». Más rasgos de esa bidireccionalidad aparecen cuando dice que la integración es un "proceso complejo, algo que se va descubriendo y haciendo, algo que se va incorporando al propio vivir, hacia dentro y hacia fuera, y eso requiere compartir significados, conceptos, objetivos, prácticas y finalidades. Algo que implica tanto al inmigrante como a la sociedad de acogida, de modo que, sin una positiva complicidad de ésta por su disposición, actitudes y capacidad de apertura hacia ellos, la integración es imposible».

Para terminar con el análisis de los diferentes modelos de integración de los planes, quedaría por definir el modelo que inspira el Plan de la Comunidad Valenciana. Este plan es exhaustivo y extenso a la hora de explicar el modelo en que se basa. Rechaza, como modelos idóneos para el contexto que trata, tanto el modelo asimilacionista francés, como el modelo «multicultural» de Gran Bretaña y Holanda. Al hablar del modelo asimilacionista y multicultural, se dice que tratan de lograr la «inserción» del inmigrante en la sociedad de acogida. El uso de este término permite definir y nombrar el modelo defendido en el plan, que viene a llamarse «modelo integracionista», es decir, basado en la integración plena del inmigrante en cada uno de los ámbitos de la vida: "Actualmente, se entiende mucho más adaptada y conveniente a la realidad social que representa el fenómeno migratorio, la aplicación de un modelo o marco para el establecimiento y el desarrollo de políticas públicas basado en la integración como instrumento que permita a las distintas instancias administrativas, gubernamentales y sociales, el establecimiento de unas pautas de convivencia común basadas en la inserción social plena de todas aquellas personas que, por unos motivos u otros, abandonan sus países de origen y emprenden la búsqueda de un país que les acoja y en el que entienden que van a poder encontrar mayores posibilidades para llevar a cabo su proyecto vital».

4. Comunicación de la Comisión al Consejo y al Parlamento Europeo sobre una política comunitaria de migración (COM (2000) 757 final) (p. 5 y 20). 
En definitiva, entre posturas polarizadas como una posible «diferenciación extrema» y un "universalismo asimilacionista» que presentaban serios inconvenientes, la búsqueda de un modelo apropiado de integración ${ }^{5}$ camina por la vía de conformarse como "la afirmación de unas identidades múltiples que supongan el desarrollo de la autonomía personal, unas identidades y lealtades de grupo etnocultural y una identidad relevante común basada en la ciudadanía compartida».

Por tanto, tras este estudio del concepto de integración que defiende cada una de las comunidades autónomas, se puede recordar la evolución de dicho concepto a la par que evoluciona el fenómeno de la inmigración en España, y ambos cambios se reflejan en las diferentes medidas que, a nivel europeo, estatal y regional, se han ido configurando. Los planes autonómicos se justifican y parten de unos principios fundacionales, así como de unas estrategias o ejes fundamentales que definen la visión que cada comunidad tiene sobre el mismo fenómeno migratorio. La razón de que haya cierto parecido entre los principios de las comunidades autónomas no es casualidad, sino fruto del seguimiento de los consejos y de las directrices marcados a un nivel superior. Por tanto, hay numerosos principios que se repiten en los diferentes planes autonómicos. Algunos de estos principios son comunes a la mayoría de los planes, mientras otros son específicos de cada uno. Aquellos principios considerados básicos, principalmente porque se mencionan en el PECI, son: bidireccionalidad, corresponsabilidad, integralidad (o globalidad), coordinación y cooperación, normalización, transversalidad, igualdad e interculturalidad.

La bidireccionalidad se refiere a una premisa sobre la que se construye el propio concepto de integración: la integración de los inmigrantes no es cosa de uno, sino de dos o más. Es decir, la integración de los inmigrantes no es responsabilidad única de los mismos inmigrantes, sino que, por el contrario, quien posee la mayor responsabilidad es la sociedad de acogida y, en concreto, las autoridades públicas, que deben fomentar la sensibilización de la población de acogida e impulsar medidas para que la acogida y la integración del inmigrante se perciba del modo más positivo posible.

En resumen, podemos concluir que el modelo de integración por el que apuestan las comunidades autónomas, a nivel teórico, se aproxima al modelo intercultural, más bien como fruto del marco de referencia y de lo correctamente político, que del análisis y las demandas de los inmigrantes. Por eso parece más adecuado utilizar el término de convivencia cívica que el de integración. Convivencia basada en el respeto mutuo y en el derecho de ciudadanía universal.

5. En concreto, el modelo de integración valenciano partiría de la definición de integración como el proceso de incorporación de los inmigrantes a la sociedad valenciana en igualdad de condiciones, derechos y obligaciones, que genera una convivencia intercultural mediante la cual puedan llegar a ser participantes activos de la sociedad, de modo que conformen, como unos valencianos más, la vida social, económica y cultural de la Comunidad Valenciana. Esta convivencia intercultural, que se trata de poner en pie entre todos, está basada en el reconocimiento de la diversidad cultural, en la interrelación y el mestizaje y en la construcción de una cultura cívica compartida, producto de la interacción y de la vida en común. 
En este sentido, es necesario dar un paso más. Así, analizada la visión de los poderes públicos sobre el concepto de integración, en este caso el de las comunidades autónomas, falta el punto de vista de los destinatarios de las políticas de integración: los inmigrantes. Este es el objetivo de los siguientes apartados, donde se analiza como los inmigrantes perciben y articulan su convivencia con los españoles. Con una conclusión clara derivada del análisis de sus discursos y que se repite en todas las comunidades autónomas analizadas (Andalucía, Cataluña, Comunidad de Madrid y Comunidad Valenciana): los usuarios de los planes de integración, los propios inmigrantes, desconocen totalmente la existencia y el contenido de dichos planes. Como punto en común entre toda la población inmigrada, los entrevistados declaran tener tres preocupaciones básicas: papeles (situación administrativa regularizada), trabajo y servicios sociales básicos (sanidad y, para aquellos con hijos, escolarización). Como se refleja en este discurso: «Los inmigrantes siempre necesitan ayuda, pero sobre todo los papeles, es lo más importante para nosotros para conseguir un trabajo. Trabajo y papeles, los inmigrantes no pueden pensar otra cosa. Nosotros queremos trabajar, siempre». En resumen, ellos demandan una política de integración distinta a la que ofrecen los poderes públicos, que se aproxima al modelo pragmático de integración apuntado por Barman y que nosotros denominamos "convivencia cívica». Por ese motivo, cualquier área que no comprenda las mencionadas estará fuera del alcance del interés de los usuarios, ya no hablando del plan en sí, que, como decimos, no es conocido por ninguno de los usuarios, sino dentro de la propia realidad cotidiana y en el entramado burocrático. Por tal motivo, sólo nos centramos en el análisis de sus preocupaciones y de sus vivencias ante el reto de conseguir regularizar su situación legal, como paso previo a todo lo demás.

\section{La integración social: «La metáfora de la camisa»}

Cuando se le preguntó a los entrevistados sobre la integración de la población inmigrada en España, las respuestas transmitieron una visión positiva y bastante clara sobre este proceso, un discurso que considera fundamental la existencia de la bidireccionalidad. La sociedad de acogida tiene también que recorrer un camino, ya que la transformación de las poblaciones es mutua. No obstante, sí es cierto que este discurso tiene algunas matizaciones por parte de algunos inmigrantes, especialmente mujeres de procedencia latina y del este de Europa, al afirmar que es más importante o necesario que el inmigrante se integre en el país, que viceversa. Discurso que refleja una percepción subjetiva y objetiva de posición social de discriminación: «La integración es de doble cara, no es de una parte solamente, lo que pasa es que los inmigrantes comprenden que están en otro país, y para que vivas mejor, tienes que hablar el idioma, más o menos, no totalmente. [...] Si tú has venido, tienes que integrarte, no son todos los nacionales los que tienen que hacer la integración» (E10), lo que destila ciertas ideas asimilacionistas sobre el modelo de integración español. 
Esta bidireccionalidad, como conformadora de la doble dirección del proceso migratorio, se muestra en ese discurso positivo de la integración: «Me considero integrada» (E6). Un ejemplo lo supone la inclusión, por parte de la población autóctona, en las redes sociales de la población inmigrada, que constituyen una parte fundamental para el óptimo desenvolvimiento de los inmigrantes en la sociedad de acogida como centros de información y contactos: "Conozco a mucha gente, pero la mayoría son españoles, y tengo muchísimos amigos, y familia que son españoles. [...] Me llevo muy bien con ellos»; «Mi experiencia ha sido buena, un $80 \%$ de lo que me he encontrado me ha recibido con los brazos abiertos, y ha hecho que me sintiese bien» (E5).

Pero la bidireccionalidad puede tener una doble acepción: entendida desde el punto de vista macro, en este caso seria una bidireccionalidad entre culturas, y desde el punto de vista micro, la bidireccionalidad seria un proceso interpersonal. Así, en la mayoría de los discursos, lo que se relata es una bidireccionalidad personal: «Yo, de los españoles, tengo buena imagen»; «Hay algunos que nos rechazan por ser inmigrantes, pero hay otros que nos acogen más que los mismos ecuatorianos» (E3); «Sí, tengo muchos amigos españoles, todas mis amigas son españolas, tengo pocas amigas rumanas» (E17). Discurso positivo que denota que es un proceso más de personas que te encuentras en el día a día, que de dialogo entre culturas que implica el modelo intercultural de integración.

En este sentido, uno de los grandes interrogantes que se plantean también con el tema de la integración de la población inmigrada es lo que ocurre con las llamadas «segundas generaciones», es decir, aquellos hijos de padres inmigrados que se han criado o han nacido en la sociedad de acogida. ¿Qué identidad cultural se les presupone a ellos? Y, entonces, ¿`se construirá una nueva identidad o, en realidad, serán identidades fragmentadas? El siguiente discurso de una mujer ecuatoriana refleja la lucha para que su hija no pierda las señas de identidad de sus raíces y, por otro lado, la importancia de la identidad de destino como proceso de no discriminación: «Mi niña nació aquí, es española, y nunca ha estado en Ecuador, o sea que se siente española. Yo le hablo de sus hermanas, [...] pero se siente de aquí. Ella quiere ir a conocer a sus hermanas, y conocer Ecuador, pero como siempre está en el cole, coge las palabras de aquí, entonces cuando habla con la hermana, empieza a usar las palabras de aquí, y las hermanas de allá no la entienden. [...] y eso que yo trato de enseñarle, pero es más de aquí que de Ecuador. Sus amigos son españoles, se lleva mejor con ellos que con los otros niños ecuatorianos. [...] Hay algunas cosas que le corrijo, pero como está aquí tiene que aprender las palabras de aquí también. No quiero liarla diciéndole cómo se dice aquí y allí. Entonces, como está en el colegio, yo tengo que seguirla a ella» (E12). La construcción de esa doble identidad, deseada más por los padres que por los hijos de las segundas generaciones, abre una ruptura en el seno de las familias inmigrantes, donde los hijos empiezan a ocupar nuevas posiciones y nuevos discursos.

La posibilidad más cercana, según los discursos analizados, es la construcción de las identidades fragmentadas en las primeras generaciones: «El inmi- 
grante tiene una vida partida. Estás pensando en lo de aquí y en lo de origen. No estás nunca cómodo. Estás bien, porque has comido, descansado, estás fresco, has luchado, pero no es eso». Ese «pero no es eso» es el reflejo más claro que justifica la existencia del propio proceso migratorio: la mejora económica, pero que revela al mismo tiempo el drama humano de un proceso de pérdida de anclaje cultural y una posición social de desigualdad en la sociedad de destino. De todas maneras, como dice Stefoni (2004): «El lugar de origen y destino se funden en una experiencia simultánea que permite la emergencia de identidades colectivas e individuales no ancladas en los tradicionales territorios, sino en espacios que se construyen día a día».

Pero hay un discurso muy crítico, sobre todo defendido por hombres con estudios medios y altos, de procedencia africana. Discurso que refleja un modelo de integración asimilacionista, muy extendido en todas las comunidades autónomas, muy crítico con el proceso de integración que tiene lugar en España. Discurso que usaba el símil de la «camisa cultural», por la que el inmigrante que llega a España ha de desprenderse de su bagaje cultural y asimilar la cultura española para que la integración en la sociedad sea efectiva: «Si tú has venido, tienes que integrarte, no son todos los nacionales los que tienen que hacer la integración, porque ellos piensan que la integración es que todo el que ha venido tiene que... Quita tu ropa y ponte la mía, come mi comida, olvida lo tuyo... Creo que hay muchos nacionales que piensan así. Pero tampoco puedes quitarte la ropa tuya, que se pongan la que ellos tienen, y si no te va, no te sientes cómodo con esa ropa, puedo ponerme hoy y mañana otra, pero la mía no la voy a tirar totalmente. En caso de que la tuya no me sirva, ¿qué voy a hacer? La mía está podrida porque la he tirado. No la puedo usar más» (E9).

Para evitar quitarse la "camisa cultural», se articula una realidad social que nada tiene que ver con la bidireccionalidad. Nos estamos refiriendo a la segregación espacial y cultural. Realidad que supone la creación de espacios separados entre ambas poblaciones, un peligro al que se está expuesto en todo momento y a lo largo de todo el proceso de integración. En este sentido, hay un discurso que afirma que la mayor parte de este peligro puede recaer en la misma población inmigrante, por una tendencia o una propensión a construir esta separación de espacios, a automarginalizarse: «Yo creo que nosotros mismos nos marginalizamos: ¿por qué nos vamos a vivir justo al lado del vecino de la misma nacionalidad? Para hacer una propia diáspora, para relacionarnos siempre los mismos, el mismo grupillo, la misma gente. ¿Qué hacemos? ¿Rechazamos convivir con la gente que estaba aquí? Y yo hablo en líneas generales. Yo no lo veo bien» (E18). Discurso que explica, en parte, el proceso de asentamiento de los inmigrantes en el espacio físico de las ciudades y los pueblos, donde las redes sociales desempeñan un papel determinante, pero también el precio de la vivienda y el rechazo de los autóctonos, lo que no seria sólo automarginación.

En definitiva, tanto si se articula un discurso positivo como negativo sobre el proceso de integración, todos comparten que lo mejor que pueden hacer los poderes públicos por la integración de los inmigrantes es ofrecer trabajo y 
que los trámites de regularización administrativa sean sencillos y rápidos, en otras palabras, conseguir de manera fácil y rápida «los papeles»: «Me considero integrada, sí. Me han ayudado, claro, lo primero con los papeles, eso ha sido el gobierno» (E2), y la cobertura indirecta que prestan otras entidades, normalmente del tercer sector: «Esa asociación tiene un papel fundamental para integrar, $[\ldots]$ porque ayudan a que todo el mundo se integre» (E10).

Pero el tema de la crisis económica, con la disminución de las ofertas de trabajo y el aumento del número de inmigrantes, ha hecho que se articule un discurso, especialmente de los que llevan más tiempo en España, donde se vislumbran las dificultades crecientes a la integración. Algunos de los entrevistados daban pistas sobre esta creciente dificultad: el aumento en el número de personas inmigradas complica la búsqueda de empleo y, en definitiva, la integración («Ahora es más difícil integrarse, antes no. Cuando llegué en 2000, no había ningún problema, podías encontrar un trabajo rápido y sin papeles, sin nada. [...] Porque en 2000 no entraba mucha gente y daban los papeles. [...] Y viene mucha gente ahora a encontrar un trabajo rápido, y ahora es muy difícil, porque hay mucha gente, no hay trabajo, y es muy difícil» (E7); «Para que la vida fuese más fácil aquí para los inmigrantes, y sea más fácil integrarse, [...] quizás el problema es que hay demasiada gente y poco trabajo» (E20). No obstante, la lectura de este hecho puede invertirse: si la población emigra hacia España, gran parte de ella lo hace confiando en que encontrará un empleo.

Sea como sea, los propios inmigrantes entrevistados sí que han puesto de manifiesto la ventaja de residir en municipios con altos porcentajes de población inmigrada, puesto que éstos cuentan con más herramientas administrativas y, sobre todo, experiencia práctica, a la hora de hacer frente al proceso de integración: «Yo me siento integrada. [...] De todos los sitios en los que he estado, aquí estoy más cómoda, no sé si por el trabajo o por todo, pero estoy mejor aquí»; "Aquí al Ayuntamiento también vine para la renovación de los papeles. Yo siempre vengo aquí cuando me hace falta»; «Vivo allí [en otro sitio], pero lo hago aquí, porque parece que allí les falta práctica, aquí se manejan mejor» (E7); «Para arreglar papeles, siempre aquí [no reside en el municipio]»; «Todo lo que he venido a solicitar aquí, me lo han arreglado y bien, además, y rápido, siempre, por eso vengo aquí» (E16).

En resumen, del análisis de los discursos de los propios inmigrantes, se desprende que ellos tienen un discurso pegado a la realidad del día a día y a la experiencia personal sobre el proceso de integración de los inmigrantes, mientras que las políticas de integración, diseñadas por las comunidades autónomas, se mueven entre la utopía política del modelo interculturalista y la realidad de las medidas del modelo asimilacionista.

\section{El reconocimiento legal: el discurso de los "papeles»}

La principal preocupación de todos los inmigrantes, a tenor de las respuestas y de los sentimientos que se desprenden de ellas, es la de tener regularizada su situación administrativa, es decir, ser «legales» en cuestión de papeles, tener 
el permiso de residencia y/o de trabajo, puesto que ser un "sin papeles», un irregular, hablando administrativamente, supone una posición de desventaja frente a los demás, además de la inseguridad propia que conlleva esta situación: «Tengo un poco de miedo, de que me agarren y me manden fuera, a mi país, aunque no haya hecho nada» (E7). Y es una de las necesidades básicas, por no decir, como los mismos entrevistados afirman, que es la necesidad a partir de la cual se estructura el resto: «Es que, en cuanto tenga papeles, puedo mejorar el empleo, buscar un buen trabajo, con un buen sueldo, conseguir una buena casa... Vivir mejor y tranquilo, no más» (E1). Cuando se alude a «vivir tranquilo», se nos está hablando, indirectamente, de la intranquilidad con que se vive antes de la regularización.

El perfil buscado de inmigrante en las entrevistas realizadas comprendía a aquellos que llevaran más de tres años en España, puesto que, a partir de ese tiempo de estancia en el país, es posible solicitar la regularización mediante el proceso denominado "de arraigo», que establece los tres años como requisito para pedir el permiso de residencia. A pesar de ello, pudieron encontrarse inmigrantes que, llevando más tiempo de los tres años establecidos, no habían tramitado aún el permiso, aunque, o bien ya se estaban informando del trámite: «No tengo permiso de residencia, no, de momento, no. Tengo pensado pedirlo»; «Estoy arreglándolo ahora, mis papeles, por arraigo, es por eso que vengo a informarme» (E10), o bien ya lo habían iniciado de hecho: "Ahora mismo no tengo el permiso de residencia, estoy en el trámite» (E3); «Ahora ya tengo contrato, y quiero conseguir mis papeles» (E8). No obstante, destacan las críticas a la situación de vacío legal que se produce hasta los tres años que se requieren para solicitar el permiso de residencia, en las que las personas se encuentran en un «limbo» administrativo, en el que residen y trabajan en el país de facto, pero no formalmente, como afirmaba esta entrevistada: «Yo sé que tener derechos implica tener deberes, tendré que pagar impuestos y esas cosas, pero es como estar en el limbo, ahorita no soy de aquí ni soy de allá, y es como que una no existe. Es todo el tema de los derechos» (E5).

Por otro lado, la mayoría de los entrevistados sí que tenían regularizada su situación administrativa, y todos ellos comentaban felizmente su experiencia una vez pasado el período de irregularidad. Las formas de conseguirlo son diversas, dependen de la manera de entrar en España: algunos llegan aquí como turistas o por carta de invitación: «Le pegué una llamada a esta amiga, y me dio una carta de invitación, y pude entrar en el país, y gracias a ella estoy aquí» (E7); otros entran a través de la contratación desde su país de origen ${ }^{6}:$ "Vine con contrato de origen como empleada doméstica, y me dieron el permiso de residencia y de trabajo»; "Yo vine con permiso directamente, porque tenía

6. Una nota característica de la comunidad autónoma catalana es un uso más intensivo de la contratación en origen, aspecto recogido en su plan de integración, y para el que se creó, desde la Generalitat de Catalunya, el Servei d'Intermediació Laboral en Origen (Servicio de Intermediación Laboral en Origen, o SILO), que asesora a las empresas interesadas y organiza este tipo de contratación dentro de un catálogo de ocupaciones de difícil cobertura. 
contrato, que me lo buscó una amiga, me envió los papeles y lo tramité en mi país» (E2). Y los que podían hacerlo, es decir, aquellos inmigrantes procedentes de países con tratado internacional, pedían la nacionalidad o la doble nacionalidad, bien antes del permiso: «No me hizo falta el permiso de residencia. Tuve la tarjeta de residencia, pero pedí la nacionalidad española por haber nacido en Barcelona» (E11); bien después de conseguirlo: «Yo tengo la nacionalidad. Primero conseguí el permiso para venir acá, y luego el contrato de trabajo». Otras opciones, que aparecieron menos frecuentemente en las entrevistas realizadas, son las de concesión del permiso por "arraigo social», es decir, una estancia prolongada de más de tres años en el país: «Sí, lo tengo. Hace poco más de un mes que lo conseguí. Lo hice por arraigo» (E7).

No obstante, para los no legales, la manera más común de normalizar su situación fue mediante las distintas regularizaciones extraordinarias realizadas por los sucesivos gobiernos de Aznar y Rodríguez Zapatero: «Cuando hubo una regularización en 2001, presenté mis documentos para pedir tarjeta de residencia» (E3); la mayoría de las cuales tuvo lugar en el 2005: "Fue cuando una regularización, creo que en el 2004 o el 2005»; "Con la regularización de 2005» (E7); «El permiso lo tengo desde la última regularización, la de 2005».

Con respecto a los trámites, la gran mayoría de los inmigrantes consultados declararon dos maneras básicas de iniciarlos: la más extendida comprende el uso de gestorías y abogados privados, mientras que la siguiente en número de usuarios responde a eso que hemos llamado «autosuficiente», es decir, la reticencia de la población inmigrada a pedir ayuda y la tendencia a resolver este tipo de asuntos por su propia cuenta y riesgo. En el siguiente discurso, se muestra la diferencia entre estos dos caminos en términos muy comprensibles y extendidos entre las variadas respuestas de la población inmigrada: «Si lo haces por tu cuenta, te sale más barato, pero tienes que perder tiempo en hacer colas, tienes que perder días de trabajo, y eso es importante» (E1). Es decir, que aquellos que acudían a gestorías privadas lo hacían más por razones de tiempo que de eficiencia o profesionalidad. $\mathrm{O}$, dicho de otra manera, si ellos mismos hubieran tenido tiempo, probablemente no habrían recurrido al sector servicios.

Las críticas al propio trámite no fueron para nada numerosas. La mayoría evaluaba el proceso burocrático como relativamente fácil, aunque las dificultades podían surgir en cualquier momento del trámite: «El trámite, la verdad, es que yo lo veo fácil, con la orientación que me han dado, lo veo fácil. Lo único más complicado es conseguir algunos papeles para el trámite» (E3). El entrevistado alude a que la mayor complicación del trámite, como afirma la mayoría de los inmigrantes, es conseguir los documentos requeridos del país de origen: «El trámite es sencillo, pero ahora se va a empezar a complicar, porque tengo que pedir cosas de mi país, y en el consulado, y voy a tener que llamar a mi hermana para que vaya al consulado de España allí, y convalide los papeles que tengo que traer, antecedentes penales y eso» (E7), algo que se complica en función del grado de burocratización de las administraciones de dichos países. 
Otras dificultades se referían a la lentitud en conseguir una cita para ser atendido, pero sobre todo encontrar un trabajo, como se comenta en el siguiente discurso: «El trámite, bueno, normal, porque había que reunir los requisitos y los papeles, no era difícil, lo difícil es conseguir la cita para que te atiendan»; o el obstáculo que suponía conseguir un contrato de trabajo: «El primer permiso, estando aquí, te daba lugar a regularizarte si estabas trabajando, era fácil, pero encontrar un trabajo para el permiso era complicado»; "Lo difícil fue conseguir una empresa que te haga el contrato de trabajo, y cuando la conseguí, ya metí mis papeles, y salió» (E7).

A pesar de ello, y como se ha señalado, la mayoría de los entrevistados percibieron el trámite como fácil: «No, esos trámites fueron fáciles, no tardan mucho, no son muy difíciles, por lo menos para los niños, no. Ha sido normal, bien»; «Fue rápido y fácil»; «El trámite para la nacionalidad lo hice yo directamente, y muy bien»; «Fue más o menos fácil»; «El trámite era fácil». También es reseñable que, como se observa en estas declaraciones, muchos de los entrevistados hicieron el papeleo por sus propios medios, informándose en algunos centros, pero realizando las gestiones ellos mismos. Sin embargo, también hay otro discurso, no mayoritario, que opina que el trámite era, en cierto modo, difícil: «Pero es un trámite complicado para el que no sabe, sí» (E9); «Hombre, era difícil porque nos lo pedían todo» (E3); «Fue difícil conseguir el permiso, porque todavía no está claro» (E1).

Otro punto a tener muy en cuenta es la importantísima ayuda que algunos empresarios prestan a la población inmigrada a su cargo en el trabajo. Hay un discurso muy extendido de los que afirman haber podido conseguir el permiso gracias a las diligencias emprendidas por sus jefes: «El trámite, como estaba trabajando, me ayudaron los jefes»; «Trabajé con un señor, $[\ldots]$ y me ayudó a hacerlo»; «El permiso para venir me lo mandó mi hermana por medio de un jefe, que me hizo los papeles, y vine a trabajar directamente como empleada doméstica» $(\mathrm{E} 1,2,7)$. Algunos, como veremos más adelante, y debido a estar trabajando, precisaron de los servicios de un abogado privado o algún tipo de gestor: «Yo, para el trámite, cogí un abogado privado, porque, como trabajaba interna, no tenía tiempo para hacerlo yo" (E15).

Preguntados los entrevistados sobre cómo se habían informado del trámite y los pasos a realizar, la población inmigrada entrevistada declaró el uso de diversos cauces. Minoritariamente, algunos entrevistados afirmaron haber llevado a cabo el proceso de forma totalmente autónoma, sin ayuda de nadie: «Lo hice todo solo. Para informarme [del estado de tramitación], a uno le daban un número del expediente, y llamaba por teléfono o iba para que me dijeran. Metí la carpeta una y otra vez, hasta que salió»; «Tengo las direcciones y voy» (E15). Otros afirmaron haberlo hecho exclusivamente por medio de un abogado, aunque lo normal es usar algún otro medio más, como veremos más adelante: «Pregunté en un abogado, pagando, y él me lo tramitó» (E10); "Antes iba a otro sitio cuando arreglaba mis papeles, a unos abogados privados, que cobraban el trámite, la consulta era gratis» (E12). 
Una buena parte de los entrevistados se ha informado del trámite gracias a sus redes sociales cercanas, como señala Soriano (2004), principalmente amigos que ya habían pasado por esa experiencia y podían ir aconsejando en cada momento del trámite: «Me informó un amigo»; «Por medio de otra persona que lo había hecho antes que yo»; «Te informas a través de tus parientes, de amigos, tus coterráneos, te enteras de la gente que ya tiene experiencia en el tema y te dicen lo que tienes que hacer» (E7).

Si las redes sociales no cumplen las expectativas, es cuando entran en juego las redes privadas, encarnadas en gestores y abogados privados ${ }^{7}$. El sector público, por tanto, parece no cumplir las expectativas ni las demandas de la población inmigrada: «Yo acudo para preguntar a un asesor con el que más o menos tengo confianza, de aquí de Cartagena, privado. Él me informa de los trámites, lo que tengo que hacer, lo que me conviene o no, cualquier cosa lo llamo a él» (E7); «No, la verdad es que no me fío. Prefiero consultar con mi abogado, que sé que está informado y que para eso le pago»; "Es que, como yo estoy trabajando, no puedo encargarme y se lo doy todo al gestor de la empresa, que se encarga de todo y me cobra poco" (E8).

La experiencia de los que han usado abogados de pago es diversa y, en función de cómo les funcionó a ellos, lo recomendarían: «Claro, contraté un abogado privado, porque otros amigos lo habían hecho antes y [...] les fue bien» (E10); «De la abogada, estoy contenta, me gusta mucho cómo trabaja. [...] Me ha ido bien mi abogado y continúo» (E2), o no: «Yo cogí una abogada, privada, de pago, [...] pero me hizo pagar y me falló en lo que hizo, no me gustó»; «Yo cogí un abogado de pago, que se encargó de todo, yo le pagué, y ya está. [...] Pero no lo haría otra vez, porque ya conozco más recursos y más sitios, ya no estoy recién venida» (E7), aunque, en principio, no se detectan demasiadas críticas negativas, como ocurría en otras comunidades.

Por otro lado, algunos de los inmigrantes ya experimentados y con bastantes años en el país conocen mejor la red de recursos legales a su alcance, por lo que han utilizado los servicios de abogados de pago y gratuitos: «Sí, me ha hecho falta. Fui a varias ONG y luego fui a un abogado privado, para resolver una consulta» (E11); "Privados también he usado, pero para otras cosas. Pero para lo de inmigración, sí he ido a varios abogados, y siempre te quedas con el que te informa mejor» (E15), dependiendo de para qué les ha hecho falta. Por lo que dejan entrever las entrevistas, para consultas van a abogados gratuitos y para emprender trámites más largos, se dirigen a los privados.

7. Una característica distintiva de la comunidad autónoma de Andalucía, según los entrevistados, es el peso de la vía privada. Hay un discurso mayoritario que afirma haber acudido a gestorías privadas para resolver el tema de la regularización administrativa: «Lo hice por una gestoría de Las Norias»; «Hay alguna que está trabajando y para no perder un día de trabajo, pues va a la gestoría»; "Sí, ya [para] la segunda [renovación] pagué en una asesoría, más rápido, no pierdes tiempo»; «Pues se va al abogado, les pide dinero, y les hace los trámites»; "Yo antes fui a una gestoría, como tienes un trabajo, no puedes parar, ni perder un día»; "Los que están trabajando, pagan en la gestoría»; «Para arreglar papeles, fui a un abogado»; «Siempre he acudido a una abogada». 


\section{El papel de las redes sociales en el proceso de integración: "a la gente que conozco»}

Como se ha podido comprobar en el apartado anterior, la opinión de los usuarios y la visión de la población inmigrada sobre la red de recursos asistenciales puesta a su disposición en todas las comunidades autónomas estudiadas y las redes sociales que cada uno de los inmigrantes posee tienen una importancia fundamental para el proceso de integración: informan de las diferentes posibilidades de ayudas económicas, son usadas para la búsqueda de empleo, dan cobertura asistencial en determinadas ocasiones (especialmente cuando hablamos de familiares que también están en España) y, en definitiva, encauzan al recién llegado, lo cual lo sitúa en el nuevo país de acogida, en lo que podría considerarse el primer paso hacia la integración real.

Por tanto, y siguiendo la tónica general observada en todas las comunidades autónomas, el inmigrante parte de un desconocimiento general de los recursos sociales disponibles a su llegada al país. Por eso, el primer contacto con las redes de ayuda es el propio entramado social cercano, aquellas personas que los acogen en el primer momento: el hermano por el que vinieron, el amigo que supieron que estaba en la ciudad, el convecino de al lado, el compatriota conocido por casualidad, el feligrés de al lado en la parroquia... Cualquiera puede ser un buen punto de información, pero también puede ser un mal punto de información.

De ahí la importancia de dichas redes sociales en el proceso de búsqueda de información para la gran mayoría de los entrevistados, que afirmaron, en los encuentros mantenidos con ellos, que serían el primer sitio donde se informarían de cualquier cosa, puesto que preguntarían a amigos, familiares, vecinos y compañeros de trabajo: «Supongo que preguntaría a mis amigos, a mi hermano»; "A la gente que conozco, no sé, amigas, las que trabajan en lo mismo que yo, vecinos»; "A mis amigas aquí, a mi compañera de piso, a mis conocidos»; "A mis amigas españolas»; "A la gente que conozco, a mis amigos españoles, a mi amiga del piso, a mi novio, que también es español, vecinos» (E16); «En fin, preguntaría a la gente, claro", posiblemente a modo de globo sonda, es decir, tanteando las diferentes posibilidades que se conocen comúnmente, a pie de calle, y antes de pasar a buscar centros específicos de información.

El segundo agente en importancia como fuente de información es el tercer sector, representado, en estas entrevistas, por las diferentes ONG y asociaciones: «Hay una asociación, [...] que me manda toda la información que necesito» (E11); "Ayer estuve en una ONG [...] para consultar» (E3). Puntos únicos de información para algunos entrevistados, así como para otros pocos, lo eran los sindicatos ${ }^{8}$ : «Me informé del trámite en el sindicato. [...] He venido otras veces aquí a informarme» (E8); "Yo sólo conozco el sindicato»; «Voy a preguntar, primero en el sindicato». Algunos, por el contrario, usan ambos puntos para

8. En la Comunidada Valenciana, al contrario que en otras comunidades como Madrid o Cataluña, pues, como refiere uno de los entrevistados, parecen ser útiles sólo en caso de conflictos laborales: «Los sindicatos sólo si ha habido algún problema de índole laboral». 
informarse: «Al sindicato, es la primera vez que he ido. He estado también en otras asociaciones»; «Yo he buscado mucha orientación aquí, y también en una ONG, y me han atendido muy bien, me han informado muy bien» (E6).

El tercer sector es, entonces, el segundo en importancia, puesto que realiza una importante labor de nexo entre las redes sociales primarias y el sector público gubernamental, donde destacan los servicios sociales municipales como representación de las entidades estatales, que ocupan el tercer puesto en las preferencias de los inmigrantes a la hora de pedir ayuda: «Sí, pregunto en Servicios Sociales, porque explicar todas las cosas, ellos me explican los sitios» (E9). Por eso, los ayuntamientos, como entes locales prestadores de servicios, tienen un peso específico en el discurso de los inmigrantes: «Preguntaría en el Ayuntamiento que me corresponde, también»; «Supongo que en el Ayuntamiento preguntaría» (E12).

Pero donde el sector público ocupa la centralidad del discurso mayoritario de los inmigrantes es en la comunidad autónoma catalana, puesto que se convierte en la segunda opción a la hora de buscar ayuda. De la misma forma que las parroquias pierden importancia en la red asistencial e informativa para la integración de inmigrantes, al tercer sector, en general, le ocurre lo mismo. Las organizaciones y asociaciones, fundamentales en otras comunidades autónomas, no funcionan de la misma manera en Cataluña, gracias a la centralización que establece el SAIER (Servicio de Atención a Inmigrantes, Extranjeros y Refugiados), que es el centro que sí tiene enorme importancia a la hora de abastecer de información a los inmigrados demandantes de la misma.

Por esta razón, el centro barcelonés conocido como SAIER es, después de las redes sociales primarias, quien articula el discurso mayoritario de los inmigrantes residentes en Cataluña: «Para aclarar dudas, lo mejor es ir al sitio [SAIER] y preguntar» (E2); "Conozco también el SAIER, que una amiga me dijo»; «También preguntaría en el SAIER»; «Pues aquí [al SAIER] había venido antes»; «También sé que puedo pasarme por el SAIER» (E20). Su importancia es fundamental para comprender la estructuración de la red de integración de inmigrantes en la comunidad catalana, puesto que este tipo de centros se encarga de organizar y estructurar toda la oferta de asistencia a inmigrantes, realizando una división de los servicios entre cada una de las asociaciones interesadas o dispuestas a prestarlos.

Por último, pero no por ello menos importante, debemos reseñar también aquí la labor, dentro de esas redes sociales cercanas tan necesarias, de las parroquias ${ }^{9}$, punto de encuentro para muchos inmigrantes con sus compatriotas y, por ende, centro de información y ayuda para la inmigración: "Al final, yo como asistía a la Iglesia de San Bastián, acudí a donde el padre, siempre a

9. Las parroquias, centros fundamentales en comunidades autónomas como Andalucía, Murcia o la Comunidad Valenciana, sin embargo, no tienen la misma relevancia en Cataluña, pues no fueron mencionadas por ninguno de los entrevistados, lo que no quiere decir que su labor sea inexistente en dicha comunidad, sino que tienen una menor participación en la red de ayuda asistencial que en otras. 
buscar faena, y hace charlas, y de ahí me salió un certificado del padre (E16); «Pues vamos a parroquias. [...] También vamos a asociaciones. Yo voy también a reuniones que hace el Padre en la Parroquia de San Vicente los domingos, hay charlas y reuniones, y también ayudan a buscar trabajo» (E17); «Pero ahora me ha dicho el Padre que no hay faena, que está complicado» (E8).

Además, cuando el inmigrante se considera informado, por propia experiencia, es cuando entonces se transforma él mismo en fuente de información para su prójimo y recomienda el recurso que a él o a ella le fue bien. Algunos de estos inmigrantes altamente experimentados se deciden a dar un paso más: se convierten en ofrecedores de información, en lugar de demandantes, y por vía oficial, ya que se deciden a crear algún tipo de asociación, sea ésta cultural, caritativa o de cualquier otro tipo, para ayudar a sus compatriotas: «Hemos formado una asociación [...]. Entonces empezamos a conocer instituciones, ONG, y a hacer proyectos, y mediante ellos conocimos a CeImigra, y nos empezaron a ayudar, en todo sentido. ACSUR-Las Segovias también nos han ayudado mucho. De momento, tenemos la organización y va funcionando. [...] Fue difícil montar la asociación» (E12).

No obstante, podemos hablar de dos perfiles diferenciados en la búsqueda de información: los aventajados y claramente experimentados, que conocen bien los recursos a su alcance, y los despistados o ajenos a la información a su disposición, que se mueven por el tejido asociativo en función de sus necesidades puntuales. Los primeros se constituyen, a su vez, en centros de información para sus compatriotas y compañeros de viaje. Los segundos seguirán dependiendo de las ayudas puntuales del momento.

Hemos seleccionado, a continuación, este extracto de una entrevista, porque es el mejor ejemplo de dicho perfil de inmigrante «experto» que menciona todas las posibilidades de ayuda que conoce y abarca la práctica totalidad del tejido asociativo del que venimos hablando. Entre paréntesis, se apunta el recurso al que se refiere, para que sean más visibles los conocimientos de los que hacía gala, inconscientemente, el entrevistado: «Pregunto a amigas (redes sociales) o a algún abogado que llamo (privado), porque conozco, porque me hizo un informe a mí, porque una amiga me recomendó. Luego llamé una vez a Tierra y Hombre, que es una ONG (tercer sector), que me informaron por teléfono, aunque me dieron informaciones contradictorias. Para aclararme, lo mejor es irme al mismo lugar y preguntar (información de primera mano). He estado muchas, muchas veces en el Ayuntamiento (gobierno municipal) para preguntar, porque mi niña tiene problemas con el lenguaje, y está en un colegio especial, y la primera vez que fui, fue para preguntar esto, me indicaron dónde tenía que ir, la trabajadora social (servicios sociales), y bien» (E16).

\section{A modo de conclusión}

Cada modelo de integración autonómico supone la aceptación de unos principios de carácter operativo y ético. Principios y modelo de integración se van conformando al mismo tiempo y son fruto de la experiencia ajena y propia en 
materia de inmigración. Además, en algunas comunidades, estos principios facilitaban la definición del modelo de integración en el que se fundamenta el plan, ya que no había referencias explícitas a un concepto de integración. Viendo las conclusiones de este estudio, se puede intuir que los principios inspiradores de los planes no van a ser muy diferentes en el núcleo central. En cambio, sí se podrán observar algunas diferencias significativas en relación con principios que podrían denominarse «coyunturales» $\mathrm{O}$ «relativos a ciertas comunidades con una tradición sociocultural propia y especialmente fuerte».

Los inmigrantes que viven en España, como les ocurre a los usuarios en general, ya sean éstos inmigrantes o autóctonos, no conocen los planes de integración autonómicos como tales, lo cual es lógico, puesto que se trata de una herramienta más administrativa que ciudadana. Los inmigrantes son parte usuaria importante de la red burocrática, inserta ésta en la red global de ayudas al ciudadano, y la mayoría conoce bien dicha red de información y servicios, una vez que se han movido alguna vez por ella, pero no conocen propiamente cómo dicha red se encuentra englobada en unos planes que tratan de ser lo más amplios posible para tratar todas las áreas potenciales de actuación, como ha podido comprobarse a lo largo de este estudio.

De las declaraciones en las diferentes entrevistas, se podría resumir que la población inmigrada, como constante general en todas las comunidades autónomas estudiadas, tiene tres preocupaciones básicas: papeles (regularidad administrativa), trabajo y servicios sociales básicos (sanidad y, para aquellos con hijos, escolarización). Todo aquello que figure en los planes y que no tenga nada que ver con dichos asuntos, queda prácticamente fuera de los intereses y, por ende, de la mayor parte de las declaraciones de los inmigrantes.

Otra constante observada en las entrevistas realizadas en las distintas comunidades autónomas es esa cierta reticencia a acudir a los servicios sociales y a las ayudas gubernamentales que los planes ponen a disposición de los inmigrantes. La población inmigrada sólo pide ayudas y subvenciones económicas cuando la situación se torna desesperada. Como ellos mismos declaran, no utilizan dichos medios porque se sirven del resto de los recursos sociales disponibles: los familiares que viven en la zona, los conocidos y amigos de la misma nacionalidad o aquellos amigos que se han hecho en España y «echan un cable» son los pilares para sobrellevar tiempos duros sin tener que recurrir a la ayuda gubernamental.

Pero esta autosuficiencia parece estar suficientemente contrarrestada con la importancia de las redes sociales para la población en general y para la población inmigrada en particular. En esa búsqueda personal, las redes sociales se configuran como el primer apoyo fundamental, y el tercer sector, como la primera ayuda externa pero más cercana. La sociedad civil ha articulado multitud de respuestas alternativas a la oficial, de modo que ha creado múltiples nodos sociales para el encuentro y el intercambio de información, como pueden ser locutorios, parroquias, varias ONG o asociaciones de inmigrantes. Las redes privadas, como los servicios jurídicos, también tienen una enorme importancia en la orientación de los recién llegados. 
Las grandes diferencias entre las distintas comunidades autónomas se establecen, en buena parte, según el grado de dependencia que tenga la población inmigrada del tejido asociativo que existe en cada región. Los inmigrantes se confiesan fieles a una ONG o a una entidad concreta cuando ésta les ha funcionado bien. La afiliación a los servicios de cualquiera de estas organizaciones depende más de las personas que trabajan en la propia organización que del servicio en sí.

Por tanto, durante el transcurso de esta investigación, se ha tenido la oportunidad de hallar dos perfiles claramente diferenciados dentro de la población inmigrada. Uno de ellos es el de gran conocedor de ayudas, mientras que el otro nos refiere a alguien indiferente a las redes asistenciales, que sólo busca trabajo y tirar para adelante. Estos perfiles dependen de muchos factores diferentes, como la edad (los más jóvenes saben moverse mejor que los más mayores, que no están tan acostumbrados a buscar burocráticamente o por Internet), la nacionalidad (aparte de la idiosincrasia propia de cada nacionalidad, está también la importante brecha que puede establecer el tema del idioma), el nivel educativo, la experiencia previa, etc.

La última dificultad que podría destacarse de las entrevistas es la que tiene que ver con la construcción de la identidad. Aunque no existe un modelo español de integración como tal, sí es cierto que los propios inmigrantes creen reconocer ciertas pautas de carácter asimilacionista. Ello se refiere, por ejemplo, al símil "cambiarse la camisa cultural», aludiendo al sentimiento personal de que la integración en España le requería este cambio de camisa para abandonar la antigua. Este símil alude a la necesidad no explícita de adaptarse al contexto nacional o autonómico, lo cual niega la existencia de la bidireccionalidad del proceso integrador, transformadora de las dos sociedades implicadas, la de llegada y la de acogida. Pero la población inmigrada no quiere perder la identidad que ya ha construido e intenta que sus hijos posean una identidad "compartida», doble, aún con el riesgo de sufrir una fragmentación. Implícitamente, también, se encuentra la visión de la inmigración como un proceso temporal y transitorio, puesto que la mayoría desea volver a sus países de origen.

En conclusión, no se desprende de la investigación realizada que, en las comunidades autónomas, exista un modelo claro para la interculturalidad, de manera que pudiera trabajarse algo tan importante y vital como es la integración de todas las personas inmigradas y de los próximos inmigrantes a nuestros sistemas sociales. Cada comunidad parece establecer sus criterios y sus prioridades en función de la realidad que vive, independientemente de la experiencia de otras comunidades.

\section{Referencias bibliográficas}

Aja, E. y Arango, J. (2009). La inmigración en tiempo de crisis. Anuario de la inmigración en España. Barcelona: Fundación CIDOB.

Aparicio, R. y Tornos, A. (2005). Las redes sociales de los inmigrantes extranjeros en España. Madrid: Ministerio de Trabajo y Asuntos Sociales. 
Arango, J. (2000). «Enfoques conceptuales y teóricos para explicar la migración». Revista Internacional de Ciencias Sociales, 165, 33-47.

Arendt, H. (1981). Los orígenes del totalitarismo. Madrid: Alianza.

- (1993): La condición humana. Barcelona: Paidós.

Ariño Villaroya, A. (2007). «Estilos de aculturación y conciencia intercultural». En: $V$ Congreso sobre la Inmigración en España. Universidad de Valencia. Celebrado entre el 21 y el 24 de marzo en Valencia. [www.adeit.uv.es/inmigracion2007]

BaUman, Z. (2001). El enigma multicultural: Un replanteamiento de las identidades nacionales, étnicas y religiosas. Madrid: Paidós.

Blanco, C. (2000). Las migraciones contemporáneas. Madrid: Alianza Ciencias Sociales.

Cornelius, W. y Lewis, J.M. (eds.) (2006). Impacts of border enforcement on Mexican Migration: The view from sending communities. Boulder CO: Lynne Rienner Publishers.

Durand, J. y Massey, D.S. (2003). Clandestinos: Migración México-Estados Unidos en los albores del siglo XXI. México D.F.: Universidad Autónoma de Zacatecas.

Huntington, S. (2004). El choque de civilizaciones. Madrid: Paidós.

IgLesias De Ussel, J. (2001). «Aspectos sociales, económicos y demográficos de la inmigración en Andalucía». En: ABad, F. y Delgado, A. Inmigración y relaciones laborales. Granada: Universidad de Granada, 11-16.

García Castaño, F.J.; Soto PaÉz, M.L. y Rubio Gómez, M. (2006). «Integración educativa». En: Barañano, A. et ali (coords.), 2006. Diccionario de relaciones interculturales. Madrid: Universidad Complutense de Madrid, 196-203

Izquierdo, A. y Noya, J. (1999). "Lugares migratorios: Una propuesta teórica y metodológica». Migraciones, 6, 19-42. Madrid: Universidad Pontificia de Comillas.

Hillmann, K-H. (2001). Diccionario enciclopédico de sociología. Barcelona: Herder.

Llopis, R. (2007). «Problemas metodológicos y epistemológicos en estudio sociológico de la inmigración de origen extranjero». Migraciones, 22, 191-220.

López Pich, P. (2007). «La política de integración de la Unión Europea». Migraciones, $22,221-256$.

Manrubia, I. (2007). «Las condiciones de acceso y ejercicio de los derechos sociales de los inmigrantes». En: Argullol i Murgadas, Enric (dir.). Inmigración y transformación social en Cataluña: Estudio jurídico comparado Barcelona: Fundación BBVA.

Montilla Martos, J.A. (2006). «Inmigración y comunidades autónomas». En: Aja, E. y Arango, J. Veinte años de inmigración en España: Perspectiva jurídica y sociológica (1985-2004). Barcelona: Fundación CIDOB, 339-398.

Pérez Yruela, M. y Rinken, S. (2005). La integración de los inmigrantes en la sociedad andaluza. Madrid: CSIC.

SARtori, G. (2006). La sociedad multiétnica: Pluralismo, multiculturalismo y extranjeros. Madrid: Taurus.

SolÉ, C. (2002). «El concepto de integración desde la sociología de las migraciones». Migraciones, 12, 9-41.

Solé, C. e IzQuierdo, A. (2005). Integraciones diferenciadas: Migraciones en Cataluña, Galicia y Andalucía, Barcelona: Anthropos.

Soriano Miras, R.M. (2010). «Análisis sociológico de la Ley 4/2000, de 11 de enero, sobre derechos y libertades de los extranjeros en España y su integración social, sus reformas $(8 / 2000 ; 11 / 2003 ; 14 / 2003 ; 2 / 2009)$ y su implicación social». Papers, 79.

- (2004). El asentamiento de la mujer marroqui en el Poniente almeriense. Madrid: Consejo Económico y Social. 
Stefoni, C. (2004). Inmigrantes transnacionales: La formación de comunidades y la transformación en ciudadanos. Chile: FLACSO. [http://bibliotecavirtual.clacso. org.ar/ar/libros/chile/flacso/artstef.pdf]

Trinidad. A. y Soriano, R.M. (2006). «Ciudadanía, política social e inmigración: El caso de las comunidades autónomas españolas». Sistema, 190-191, 105-142.

Trinidad, A. y Alemán, C. (2006). Servicios sociales: Planificación y evaluación. Navarra: Thomson-Civitas, Aranzadi.

Trinidad, A.; Carrero, V. y Soriano, R.M. (2006). Teoria fundamentada "Grounded Theory": La construcción de la teoría a través del análisis interpretacional. Madrid: CIS. Cuadernos Metodológicos; 37.

Zapata-BArrero, R. (2003). «La ciudadanía en contextos de multiculturalidad: procesos de cambios de paradigmas». Anales de la Cátedra Francisco Suárez, 37, 173-199.

- (2004). Multiculturalidad e inmigración. Madrid: Síntesis.

Planes autonómicos sobre integración de inmigrantes

Andalucía. Consejería de Gobernación (2006). II Plan Integral para la Inmigración en Andalucía 2006-2009. Sevilla: Consejería de Gobernación.

Cataluña. Secretaria per a la Immigració (2005). Pla de Ciutadania i Immigració de Cataluña, 2005-2008. Barcelona: Departament de Benestar i Família. Secretaria per a la Immigració.

Comunidad de Madrid. Consejería de Inmigración (2006). Plan de Integración 2006-2008. Madrid: Consejería de Inmigración.

Comunidad Valenciana. Dirección General de Inmigración (2004). Plan Valenciano de Inmigración 2004-2007. Valencia: DGI.

Región de Murcia. Dirección General de Política Social (2002). Plan para la Integración Social de los Inmigrantes de la Región de Murcia, 2002-2004. Murcia: Consejería de Trabajo y Política Social. 